\title{
Attentional bias modulation by reappraisal in patients with generalized anxiety disorder: an event-related potential study
}

\author{
H.Y. $\operatorname{Han}^{1}$, T. Gan ${ }^{2,3}$, P. Li ${ }^{4}$, Z.J. Li ${ }^{1,5}$, M. Guo ${ }^{1}$ and S.M. Yao ${ }^{1}$ \\ ${ }^{1}$ Beijing Anding Hospital, Capital Medical University, Beijing, China \\ ${ }^{2}$ Department of Psychology, Zhejiang Sci-Tech University, Hangzhou, China \\ ${ }^{3}$ State Key Laboratory of Cognitive Neurosciences and Learning, Beijing Normal University, Beijing, China \\ ${ }^{4}$ Department of Psychiatry, Qiqihaer Medical University, Qiqihaer, Heilongjiang, China \\ ${ }^{5}$ Department of Psychiatry and Clinical Psychology, Capital Medical University, Beijing, China
}

\begin{abstract}
Affective states influence subsequent attention allocation. We evaluated emotional negativity bias modulation by reappraisal in patients with generalized anxiety disorder (GAD) relative to normal controls. Event-related potential (ERP) recordings were obtained, and changes in P200 and P300 amplitudes in response to negative or neutral words were noted after decreasing negative emotion or establishing a neutral condition. We found that in GAD patients only, the mean P200 amplitude after negative word presentation was much higher than after the presentation of neutral words. In normal controls, after downregulation of negative emotion, the mean P300 amplitude in response to negative words was much lower than after neutral words, and this was significant in both the left and right regions. In GAD patients, the negative bias remained prominent and was not affected by reappraisal at the early stage. Reappraisal was observed to have a lateralized effect at the late stage.
\end{abstract}

Key words: Reappraisal; Generalized anxiety disorder; Event-related potential; P200; P300

\section{Introduction}

Patients with generalized anxiety disorder (GAD) are characterized by emotional hyperarousal, and their negative emotions are more intense than those of most other people (1). This emotional dysfunction in patients with GAD can result in attentional bias. Studies have shown that individuals with GAD experience an attention bias toward threat (2-4), which results in the hyperarousal of negative emotions. Furthermore, research has indicated that the effects of cognitive bias in anxious persons may depend on their mood (5). However, it is not known whether attentional bias is modulated in GAD patients after their mood is improved.

According to Gross' emotion regulation theory, the cognitive strategy of reappraisal can reduce behavioral and subjective signs of disgust and anxiety $(6,7)$. Reappraisal can successfully up- or downregulate negative emotions (8-10). Blair et al. (11) found that during emotional regulation testing, GAD patients exhibited reduced blood oxygen level-dependent responses in areas of the brain implicated in top-down attentional control. This suggests that reappraisal may change emotion. If GAD patients utilize reappraisal to downregulate negative emotions, the amount of attention they allocate to negative stimuli probably would be reduced.

Event-related potential (ERP) waveforms reflect the brain's response to specific events and are very useful for assessing attentional bias to emotional stimuli. They have been extensively applied to the study of attention to emotional stimuli such as fear (12), anger (13), and emotion-word Stroop effect (14), and have been effective in other psychological and cognitive studies $(15,16)$. Attention bias is preferentially sustained by affective significance stimuli (17), which can be recorded by P200 and P300 registered in the frontal and parietal areas of the brain. For example, the positive peak occurring $\sim 200 \mathrm{~ms}$ after initiation of a stimulus (P200) is particularly involved in negative attentional bias, and amplitudes at this peak are higher in response to negative stimuli than to positive ones (18). The P300 of the ERP has also been used extensively to study emotional processing and attentional bias. Both

Correspondence: Z.J. Li, Department of Psychiatry and Clinical Psychology, Capital Medical University, Beijing 100088, China. Fax: +86-10-5830-3004. E-mail: lizhj8@ccmu.edu.cn 
pleasant and unpleasant words can evoke a higher P300 than neutral words, indicating that greater attention resources are allocated to emotive materials (19). In healthy participants, P300 amplitudes decreased with increasing unpleasant emotions by reappraisal $(10,20,21)$. Thus, regulating negative emotions by reappraisal, attention allocation changes are reflected by the P300 amplitude. However, no research has focused on changes in P200 and P300 amplitudes in patients with GAD when unpleasant emotions are decreased.

For the present study, we hypothesized that because GAD patients have less ability to regulate emotion, and because that can influence subsequent attention allocation, P200 and P300 amplitudes should be higher in GAD patients than normal controls after decreasing negative emotion by reappraisal. This study will help to understand the character of attentional bias modulation in patients with GAD at the early and late stages of the reappraisal process.

\section{Material and Methods}

\section{Participants}

The Institutional Review Board of Beijing Anding Hospital approved this study. All GAD patients were recruited at the Beijing Anding Hospital. Diagnoses were established according to the Anxiety Disorders Interview Schedule for DSM-IV: Lifetime version (22). Exclusion criteria were the presence of a diagnosis other than GAD as a primary disorder on axis I, or any other diagnosed problems on axes II, III, or IV of the DSM-IV. GAD patients currently receiving any form of behavioral, cognitive, or medication treatments were excluded. None of the control patients had been diagnosed with psychiatric or neurological disease.

Sixteen GAD patients (8 men, 8 women with a mean age of $31.56 \pm 7.53$ years) were recruited. The total Hamilton Anxiety Rating Scale score was $20.94 \pm 5.50$, and the mean education level was $15.00 \pm 2.93$ years. Seventeen healthy control participants ( 8 men, 9 women) were recruited following a request for individuals between 18 and 50 years of age. The mean age of this group was $32.75 \pm 8.36$ years, and there were no differences between the GAD patients and controls in age or education level ( $P>0.05$ for both). Prior to testing, every participant was familiarized with the laboratory setting, and the experiment was explained to each in general terms. All participants signed an informed consent form.

\section{Stimuli}

All subjects in the GAD patient and control groups were given two types of stimuli, pictures and words. A Likert scale, ranging from 1 to 9 , was used to evaluate both valence and arousal of the pictures and words. The pictures consisted of 80 unpleasant images (mean $\pm S D$, valence $=2.68 \pm$ 0.53 ; arousal $=5.90 \pm 0.59$ ) and 80 neutral images (valence $=5.13 \pm 0.28$; arousal $=4.18 \pm 0.77$ ) drawn from the Chinese affective picture system (23). Word stimuli (each word including 2 characters) consisted of 80 negative (valence $=2.80 \pm 0.29$; arousal $=5.82 \pm 0.47$ ) and 80 neutral words (valence $=5.08 \pm 0.16$; arousal $=4.40 \pm 0.82$ ) drawn from the Chinese affective words system (24). Each picture and word was presented only once in each block. The 160 trial pictures and words were divided into four blocks for use in the investigation.

The 40 trials in each block were presented in four sets of 10 image-word combinations, with 10 trials for each condition. Each set consisted of one of 4 possible conditions (below), while each image-word combination was presented randomly (10). In each trial, the subject was first exposed to an image (neutral or negative), and then a word (neutral or negative). The possible combinations (i.e., conditions) were designated $S_{\text {neutral/neutral, }}$ $S_{\text {neutral/negative, }} S_{\text {negative/neutral, and }} S_{\text {negative/negative, }}$ respectively. The initial exposure to a neutral or unpleasant image meant that the subject's response to the following word would occur after either a neutral condition had been established, or a negative emotion had been downregulated, respectively.

\section{Emotion regulation (ER) instructions}

The reappraisal emotion regulation strategy of suppressing emotion (10) was employed. Participants were instructed to imagine that the situation depicted in the picture was not real, but rather that it was part of a movie, or just a picture. By thinking in this way, negative emotions in response to a picture are decreased. Participants were also instructed to regulate their emotions towards both negative pictures and neutral pictures by reappraisal of the pictures. All of the participants were given ER instructions for 20-30 min until they understood them.

\section{Procedure}

The task was based on work previously described by Deveney and Pizzagalli (10). During the task, participants were instructed to passively view the picture during the first $5 \mathrm{~s}$, during which no suppression by reappraisal was required. After the initial $5 \mathrm{~s}$, an automated voice instructing "emotional regulation" in Chinese was given through a set of speakers above the monitor. This voice instructed participants to suppress their emotional experience using the reappraisal ER strategy detailed above. The ER phase lasted for $5 \mathrm{~s}$, and at the same time the picture disappeared from the screen. Following a 600-ms delay a Chinese word appeared in the center of the screen. The participants were asked to identify whether the word was negative (by pressing ' 1 ' on the keyboard) or neutral (pressing ' 2 ' on the keyboard) and ERPs were recorded simultaneously. The words disappeared when the participants pressed the button. The presentation of the next trial followed the response to the word with an interval of $4 \mathrm{~s}$ (Figure 1). 


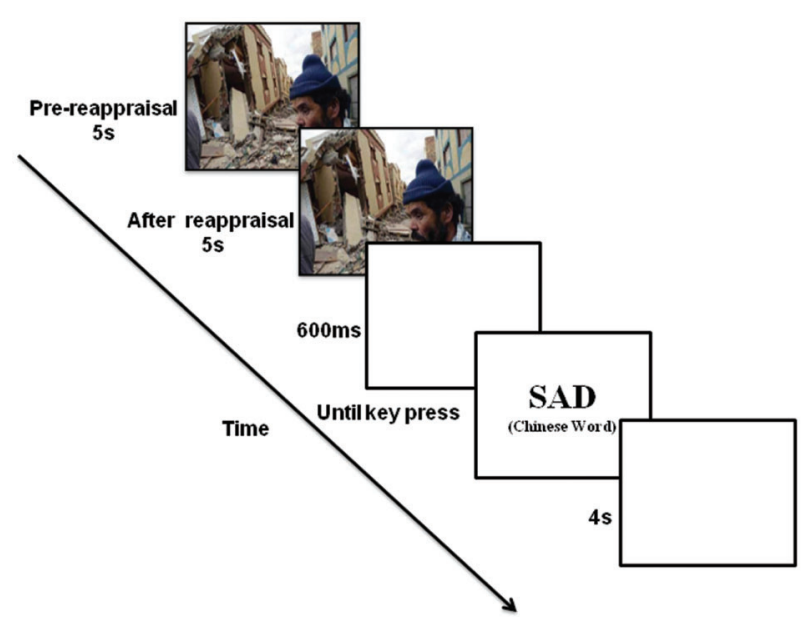

Figure 1. Reappraisal task procedure. Participants viewed the pictures for $5 \mathrm{~s}$, and then they reappraised them. After a 5-s regulation period and a short delay, a negative or neutral word appeared. Participants were asked to press response buttons to indicate whether the word was negative or neutral. Reaction time, $\mathrm{P} 200$, and P300 were measured in response to the negative and neutral words.

Initially, five trials were presented randomly for the purpose of practice, after which participants progressed through the four blocks in order. Upon completion of each block, the participants were given a rest break and instructed to breathe lightly, relax, and close their eyes. All the participants used their right hands to make each word judgment by pressing 1 for the negative words and 2 for the neutral words.

After listening to the instructions, participants were given the chance to ask questions and were provided with additional examples, until the experimenter felt that each of them fully understood the emotion regulation instructions. As an additional manipulation check, the experimenter reviewed the participants' responses to the questionnaire after the experiment to determine whether they understood the instructions and routinely used the reappraisal strategy (25).

\section{ERP recording and data analysis}

Electroencephalograms (EEGs) were recorded from 64 scalp sites using electrodes mounted on an elastic cap (Brain Product, $\mathrm{GmbH}$, Germany), with an online reference to the left mastoid. The horizontal electro-oculogram (HEOG) was recorded using two electrodes placed lateral to the right and left eyes. The vertical electro-oculogram (VEOG) was recorded with electrodes placed above and below the right eye. All interelectrode impedances were maintained below $5 \mathrm{k} \Omega$. The EEG and electro-oculogram (EOG) were amplified using a $0.01-100 \mathrm{~Hz}$ band-pass filter and continuously sampled at $500 \mathrm{~Hz}$ in each channel. For off-line analysis, all EEG signals were rereferenced to the average of the left and right mastoids.
A linear interpolation was used to correct for corrupted channels (26). The EEG data were low-pass-filtered below $50 \mathrm{~Hz}$ (24 dB/oct) and were corrected for eye movements or blinks with the Gratton and Coles' method as implemented in the Brain Vision analysis software (Brain Product, $\mathrm{GmbH}$ ). Trials containing EEG sweeps with amplitudes exceeding $\pm 100 \mathrm{mV}$ were excluded. In each condition, no subject had more than 10 unacceptable trials.

The ERP waveforms were time-locked to the onset of the word stimulus; the average epoch was $1800 \mathrm{~ms}$, including a $200 \mathrm{~ms}$ pre-stimulus baseline. We recorded the amplitudes of P200 and P300 and calculated their mean values. Electrode sites were chosen based on previous studies (27) and the visual inspection of the ERP grand average waveforms. For P200, mean amplitudes from 160 to $230 \mathrm{~ms}$ were recorded from electrodes placed at F1, F3, F5 (right anterior), and F2, F4, F6 (left anterior). For P300, mean amplitudes from 280 to $380 \mathrm{~ms}$ were analyzed and 24 electrodes placed anterior to posterior for left (F1, F3, F5, FC1, FC3, FC5, CP1, CP3, CP5, P1, $\mathrm{P} 3, \mathrm{P} 5)$ and right (F2, F4, F6, FC2, FC4, FC6, CP2, CP4, $\mathrm{CP} 6, \mathrm{P} 2, \mathrm{P} 4, \mathrm{P} 6$ ) regions: left and right were selected for this component.

The following within-subject factors were used in the analysis: region (left or right), picture valence (negative or neutral), and word valence (negative or neutral). Repeated analyses of variance (ANOVA) were performed with the factors group (GAD or control), picture valence (positive or negative), and word valence. GreenhouseGeisser corrections were adopted wherever appropriate.

Reaction time data were used for data analysis. These were averaged across conditions for each participant and submitted to separate (GAD, control) $\times 2$, (negative, neutral pictures) $\times 2$, (negative, neutral words) ANOVAs. SPSS 16.0 was used for all statistical analyses (IBM, USA). $\mathrm{P}<0.05$ was considered to be significant.

\section{Results}

The reaction times of the GAD patients and normal controls differed significantly, as indicated by the interaction of word valence $\times$ group $[F(1,31)=4.731, P=0.037$, $\left.\eta^{2}=0.132\right]$, and post hoc Neuman-Keuls comparisons indicated that GAD patients responded more slowly to neutral words than did the normal controls $(P=0.030$; Figure 2, Table 1).

For the mean anterior P200 amplitude, picture and word valence were identified as the main effectors $\left(F(1,31)=15.98, P=0.000, \eta^{2}=0.340\right)$ and $(F(1,31)=6.95$, $\left.P=0.013, \eta^{2}=0.183\right)$, respectively. Most important, the interaction of word valence $\times$ group was significant $\left(F(1,31)=4.48, P=0.038, \eta^{2}=0.131\right)$. In GAD patients only, the mean P200 amplitudes in response to negative words were significantly higher than the mean P200 amplitudes in response to neutral words $(P=0.002$; 


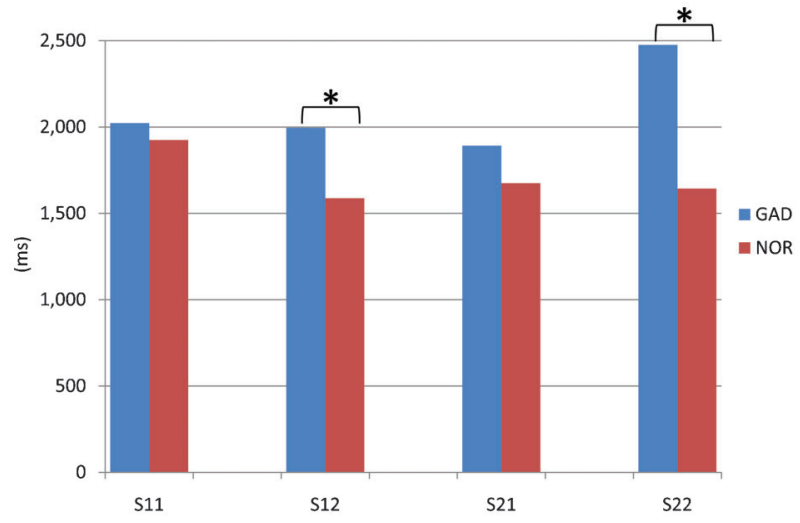

Figure 2. Comparison of reaction time under $S_{\text {negative/negative, }}$ $S_{\text {negative/neutral }}, S_{\text {neutral/negative, and }} S_{\text {neutral/neutral }}$ conditions between patients with generalized anxiety disorder (GAD) and normal controls. ${ }^{*} \mathrm{P}<0.05$, post hoc Neuman-Keuls comparisons test.

Figure 3, Table 2). The P200 amplitude was not significantly affected by any other stimulus or interaction of stimuli.

For the mean P300 amplitude, word valence was revealed as a significant effect. The mean P300 amplitudes in response to negative words were higher than the mean P300 amplitudes in response to neutral words $\left(F(1,31)=8.39, P=0.007, \eta^{2}=0.213\right)$. In addition, the interaction of picture valence $\times$ word valence $(F(1,31)=$ $\left.7.47, P=0.010, \eta^{2}=0.194\right)$ and region $\times$ picture valence $\times$ word valence $\times$ group $\left(F(1,31)=5.41, P=0.027, \eta^{2}=0.116\right)$ significantly affected the mean P300. Post-hoc comparisons indicated that for the GAD patient group, there was a mean P300 amplitude response of $S_{\text {negative/negative }}<S_{\text {neutral/negative }}$ $(P=0.016)$ only in the right region. However, for the normal control group, there was a significant effect of $\mathrm{S}_{\text {negative/negative }}<\mathrm{S}_{\text {neutral/negative }}$ in the left and right regions. For the normal control group, there was a significant effect of $S_{\text {negative/negative }}<S_{\text {neutral/negative }}(P=0.021$ for left region and $\mathrm{P}=0.022$ for right region, respectively). Moreover, for the GAD patient group, there was a higher mean P300 amplitude in response to negative words than to neutral words after neutral pictures $\left(S_{\text {neutral/negative }}>S_{\text {neutral/neutral; }}\right.$; $\mathrm{P}=0.002$ and 0.001 ), which was not seen in the normal group. There was no significant difference in the conditions $S_{\text {negative/neutral }}$ and $S_{\text {neutral/neutral }}(P>0.05)$ between the groups (Figure 4, Table 3).

\section{Discussion}

GAD patients experience an attention bias toward threat that results in the hyperarousal of negative emotions. We investigated whether this bias, after decreasing negative emotion through conscious reappraisal, would be reflected by differences in P200 and P300 amplitudes relative to normal controls. We reasoned that such differentials would exist, and our data bear this out. After training in ER, the subjects were presented with either a neutral or unpleasant image for $5 \mathrm{~s}$ and instructed to reappraise and downregulate negative emotions. After $5 \mathrm{~s}$ of exposure to the image and $5 \mathrm{~s}$ of reappraisal, the image was replaced with a neutral or negative word after a 600-ms delay. Participants judged whether the word was negative or neutral, ERPs (P200 and P300) were recorded, and their choice was timed. Patients with GAD spent more time than normal controls judging neutral information. Furthermore, only in the GAD group was the P200 amplitude in response to negative words higher than the P200 amplitude in response to neutral words. This suggests that the greater sensitivity to negative stimuli in GAD patients than in the normal controls was reflected by the higher P200 amplitude. In both groups, after downregulation of negative emotion, the mean P300 amplitude was much lower in response to negative than to neutral words. However, in GAD patients, this response was significant only on the right, whereas in normal controls the response was bilateral.

The results of our study suggested that the reaction time was not affected by a prior reappraisal process in either GAD patients or normal controls. This result is consistent with Deveney and Pizzagalli's study (10) in which behavioral performance was not changed by reappraisal regulation. Furthermore, in the present study, patients with GAD spent more time than normal controls judging neutral information.

In a modified version of the Go/No go task, it was reported that extended reaction times to Go cues (letter "M") were longer during the mental processing of negative or neutral contexts than during positive contexts (28). Therefore in our study, the behavioral performance result may indicate that GAD patients are less able to deal

Table 1. Reaction times under each condition (ms).

\begin{tabular}{|c|c|c|c|}
\hline & Image and word valence & GAD & Control \\
\hline$S_{\text {neutral/neutral }}$ & Image neutral/word neutral & $2.48 \pm 1.44$ & $1.63 \pm 0.50$ \\
\hline$S_{\text {neutral/negative }}$ & Image neutral/word negative & $1.89 \pm 0.63$ & $1.66 \pm 0.53$ \\
\hline$S_{\text {negative/neutral }}$ & Image negative/word neutral & $2.00 \pm 0.93$ & $1.57 \pm 0.52$ \\
\hline$S_{\text {negative/negative }}$ & Image negative/word negative & $2.02 \pm 0.58$ & $1.89 \pm 0.70$ \\
\hline
\end{tabular}

Data are reported as means \pm SD. GAD: generalized anxiety disorder. 

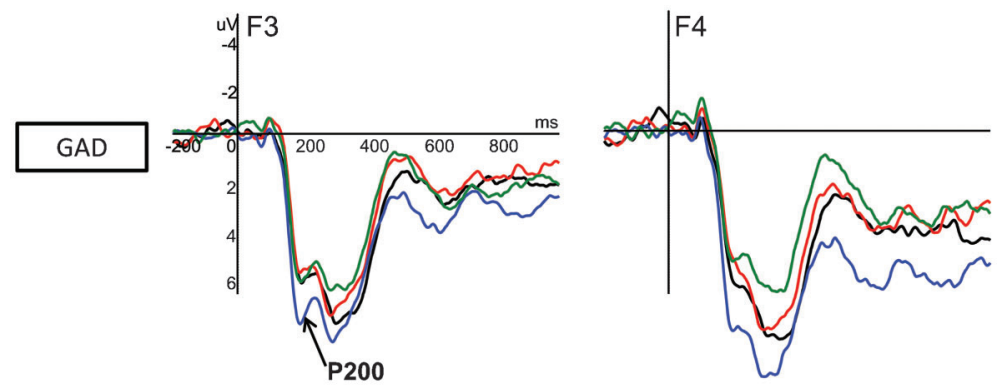

Figure 3. Event-related potentials recorded for patients with generalized anxiety disorder (GAD) and normal controls under $S_{\text {negative/negative, }}$ $\mathrm{S}_{\text {negative/neutral, }}, \mathrm{S}_{\text {neutral/negative }}$ and $\mathrm{S}_{\text {neutral/neutral }}$ conditions, showing P200 amplitude at the site of F3 and F4.

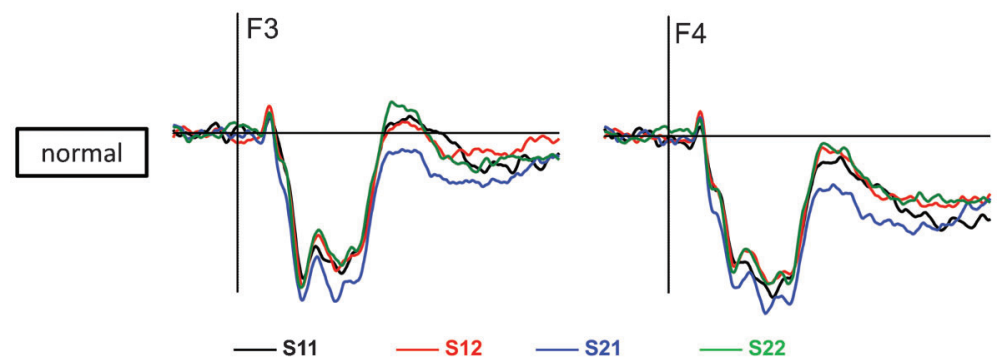

not change at the early stage of perception after reappraisal. There has been research showing that biased attention might be inherent in anxiety disorder (31) and preconscious biases of attention toward a negative stimulus heightened vulnerability to anxiety (32). Our observations in this study indicate that downregulating negative emotion by reappraisal in GAD patients will not affect the early stage of attention to negative information.

For GAD patients, the P300 amplitudes were higher in response to $S_{\text {neutral/negative }}$ trials than they were to the $S_{\text {neutral/neutral }}$ trials, but in the normal controls the P300 amplitudes were not significantly different. This indicated that only in the GAD group was the P300 amplitude in response to negative words significantly higher than to neutral words under neutral conditions. This is consistent with a previous study showing that in GAD patients, emotional words could evoke more positive P300 amplitudes than neutral words (19). Our results are also consistent with a previous report that GAD patients showed more emotion negativity bias than did normal controls (33).

Table 2. Mean amplitudes for P200 on the left and right sides under each condition $(\mu \mathrm{v})$.

\begin{tabular}{llccccc}
\hline & Image and word valence & \multicolumn{2}{c}{ GAD } & & \multicolumn{2}{c}{ Control } \\
\cline { 3 - 5 } \cline { 5 - 6 } & & Left & Right & & Left & Right \\
\hline$S_{\text {neutral/neutral }}$ & Image neutral/word neutral & $5.94 \pm 3.19$ & $5.29 \pm 2.88$ & & $5.36 \pm 1.85$ & $4.96 \pm 2.19$ \\
$S_{\text {neutral/negative }}$ & Image neutral/word negative & $7.20 \pm 3.10$ & $6.28 \pm 2.80$ & & $5.95 \pm 2.23$ & $5.30 \pm 1.99$ \\
$S_{\text {negative/neutral }}$ & Image negative/word neutral & $5.27 \pm 2.61$ & $4.79 \pm 2.05$ & & $5.27 \pm 2.29$ & $4.96 \pm 2.15$ \\
$S_{\text {negative/negative }}$ & Image negative/word negative & $5.95 \pm 3.57$ & $5.74 \pm 3.09$ & & $4.97 \pm 1.83$ & $4.71 \pm 2.03$ \\
\hline
\end{tabular}

Data are reported as means \pm SD. GAD: generalized anxiety disorder. 


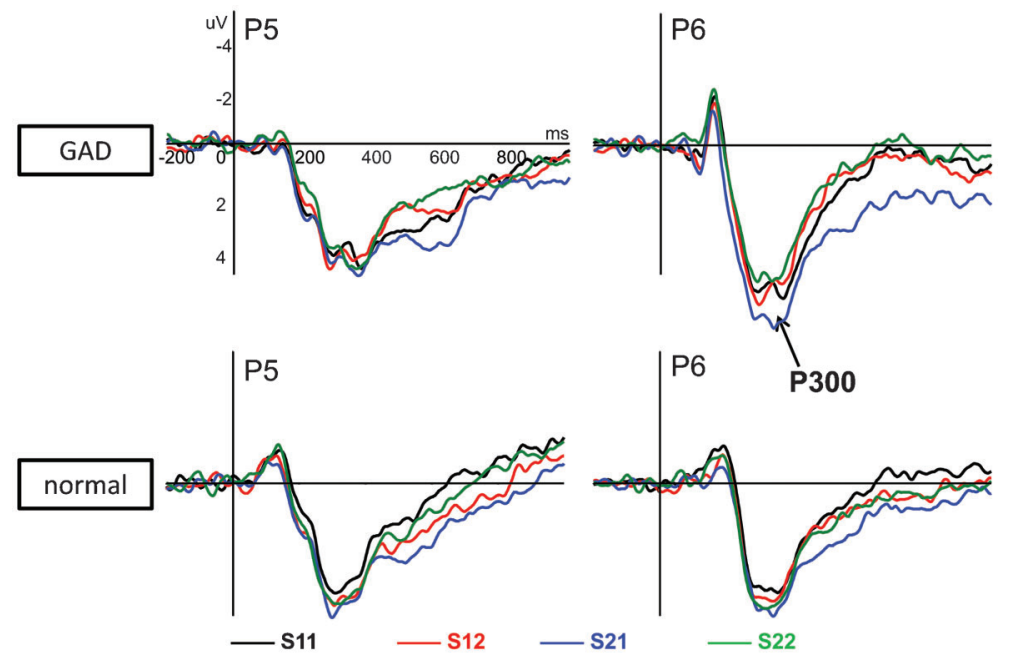

Figure 4. Event-related potentials recorded for patients with generalized anxiety disorder (GAD) and normal controls under $S_{\text {negative/negative, }}$ $S_{\text {negative/neutral, }}, S_{\text {neutral/negative }}$ and $S_{\text {neutral/neutral }}$ conditions, showing P300 amplitude at the site of P5 and P6.
However, our results are inconsistent with the findings of Deveny and Pizzagalli (10). In their study, the P300 amplitudes of normal individuals in response to a decreasing unpleasant condition were similar to those in response to a neutral condition. In our study, for both GAD patients and controls, after negative emotions were downregulated the P300 amplitudes in response to negative words were lower than for the neutral condition $\left(S_{\text {negative/negative }}<\right.$

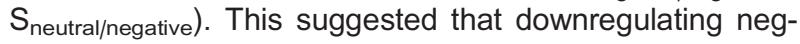
ative emotion may modulate the cognitive resource allocation under a negative stimulus, both in normal controls and GAD patients. Specifically, this effect on the P300 amplitude was significant only at the right region for GAD patients, but was significant at both hemispheres for the control group. The lack of interaction effect at the left region may reveal a weakness in late-stage emotion processing in GAD patients. Patients with GAD have difficulties in modulating negative emotions and using fixed cognitive control strategies to avoid some experiences (34). Our results indicated that these difficulties in GAD patients could be reflected by the P 300 .

In GAD patients, the right hemisphere downregulation of negative emotion caused a subsequent lowering of P300 amplitude in response to negative words, compared with the neutral condition. Research found that anxietyprone individuals engage prefrontal cortical systems more when downregulating emotions. Compared to controls, anxious participants displayed greater left dorsolateral prefrontal cortical activity during downregulation of emotion (35). Thus, there is a lateralized effect when downregulating emotions in anxiety-prone persons. Our current findings are in agreement with this. However, in GAD patients, this effect of negative words after downregulation is significant only on the right at the late stage (P300).

The current study could be considered limited by the relatively small sample size, and further studies are

Table 3. Mean amplitudes for $\mathrm{P} 300$ in four brain regions under four conditions $(\mu \mathrm{v})$.

\begin{tabular}{|c|c|c|c|c|c|}
\hline & \multirow[t]{2}{*}{ Image and word valence } & \multicolumn{4}{|c|}{ Region } \\
\hline & & 1 & 2 & 3 & 4 \\
\hline \multicolumn{6}{|l|}{ GAD } \\
\hline$S_{\text {neutral/neutral }}$ & Image neutral/word neutral & $6.38 \pm 2.42$ & $6.87 \pm 3.01$ & $6.01 \pm 2.70$ & $6.87 \pm 2.25$ \\
\hline$S_{\text {neutral/negative }}$ & Image neutral/word negative & $7.92 \pm 3.04$ & $8.59 \pm 3.84$ & $7.17 \pm 3.76$ & $8.28 \pm 3.63$ \\
\hline$S_{\text {negative/neutral }}$ & Image negative/word neutral & $7.09 \pm 3.32$ & $7.86 \pm 3.86$ & $5.92 \pm 3.47$ & $7.15 \pm 2.90$ \\
\hline$S_{\text {negative/negative }}$ & Image negative/word negative & $7.50 \pm 2.73$ & $6.50 \pm 3.87$ & $6.22 \pm 3.11$ & $6.91 \pm 2.71$ \\
\hline \multicolumn{6}{|l|}{ Control } \\
\hline$S_{\text {neutral/neutral }}$ & Image neutral/word neutral & $5.94 \pm 3.47$ & $6.45 \pm 3.61$ & $6.19 \pm 3.25$ & $6.45 \pm 2.91$ \\
\hline$S_{\text {neutral/negative }}$ & Image neutral/word negative & $6.87 \pm 3.57$ & $7.12 \pm 3.71$ & $6.59 \pm 3.76$ & $7.05 \pm 3.19$ \\
\hline$S_{\text {negative/neutral }}$ & Image negative/word neutral & $6.62 \pm 3.56$ & $6.85 \pm 3.71$ & $5.89 \pm 3.03$ & $6.10 \pm 2.74$ \\
\hline $\mathrm{S}_{\text {negative/negative }}$ & Image negative/word negative & $5.97 \pm 3.51$ & $6.50 \pm 3.86$ & $5.62 \pm 2.66$ & $6.01 \pm 2.70$ \\
\hline
\end{tabular}

Data are reported as means \pm SD. GAD: generalized anxiety disorder. Region 1: F1, F3, F5, FC1, FC3, FC5; region 2: F2, F4, F6, FC2, FC4, FC6; region 3: CP1, CP3, CP5, P1, P3, P5; region 4: CP2, CP4, CP6, P2, P4, P6. 
needed to corroborate and build upon these findings. In addition, the lack of physiological recordings was a limitation, and in future studies these will be added during reappraisal.

In summary, in GAD patients after downregulation of negative emotion by reappraisal, the modulation of emotional negativity bias followed a temporal course. At the early stage, the negative bias remained prominent and was not affected by reappraisal. However, at the late stage the reappraisal was observed to have a lateralized effect. This may constitute an inherent phenotype of GAD

\section{References}

1. Turk CL, Heimberg RG, Luterek JA, Mennin DS, Fresco DM. Emotion dysregulation in generalized anxiety disorder: A comparison with social anxiety disorder. Cognitive Therapy and Research 2005; 29: 89-106, doi: 10.1007/ s10608-005-1651-1.

2. Mathews A, MacLeod C. Selective processing of threat cues in anxiety states. Behav Res Ther 1985; 23: 563-569, doi: 10.1016/0005-7967(85)90104-4.

3. Bar-Haim Y, Lamy D, Pergamin L, Bakermans-Kranenburg $\mathrm{MJ}$, van IJzendoorn MH. Threat-related attentional bias in anxious and nonanxious individuals: a meta-analytic study. Psychol Bull 2007; 133: 1-24, doi: 10.1037/0033-2909. 133.1.1.

4. Amir N, Beard C, Burns M, Bomyea J. Attention modification program in individuals with generalized anxiety disorder. J Abnorm Psychol 2009; 118: 28-33, doi: 10.1037/a0012589.

5. Mathews A, Mogg K, Kentish J, Eysenck M. Effect of psychological treatment on cognitive bias in generalized anxiety disorder. Behav Res Ther 1995; 33: 293-303, doi: 10.1016/0005-7967(94)E0022-B.

6. Gross JJ. Antecedent- and response-focused emotion regulation: divergent consequences for experience, expression, and physiology. J Pers Soc Psychol 1998; 74: 224237, doi: 10.1037/0022-3514.74.1.224.

7. Hofmann SG, Heering S, Sawyer AT, Asnaani A. How to handle anxiety: The effects of reappraisal, acceptance, and suppression strategies on anxious arousal. Behav Res Ther 2009; 47: 389-394, doi: 10.1016/j.brat.2009.02.010.

8. Jackson DC, Malmstadt JR, Larson CL, Davidson RJ. Suppression and enhancement of emotional responses to unpleasant pictures. Psychophysiology 2000; 37: 515-522, doi: 10.1111/1469-8986.3740515.

9. Ochsner KN, Ray RD, Cooper JC, Robertson ER, Chopra S, Gabrieli JD, et al. For better or for worse: neural systems supporting the cognitive down- and up-regulation of negative emotion. Neuroimage 2004; 23: 483-499, doi: 10.1016/j.neuroimage.2004.06.030

10. Deveney CM, Pizzagalli DA. The cognitive consequences of emotion regulation: an ERP investigation. Psychophysiology 2008; 45: 435-444, doi: 10.1111/j.1469-8986.2007.00641.x.

11. Blair KS, Geraci M, Smith BW, Hollon N, DeVido J, Otero M, et al. Reduced dorsal anterior cingulate cortical activity during emotional regulation and top-down attentional control in generalized social phobia, generalized anxiety disorder, and comorbid generalized social phobia/generalized anxiety patients, in that cognitive resource allocation is lateralized and response is delayed.

\section{Acknowledgments}

Research supported in part by the projects of the Ministry of Science and Technology of the People's Republic of China: the Eleventh Five-year National Key Technology R\&D Program (Grant No. 2009BAI77B11) and the Capital Health Development Research Projects (\#2011-2012-05).

disorder. Biol Psychiatry 2012; 72: 476-482, doi: 10.1016/ j.biopsych.2012.04.013

12. Yang J, Yuan J, Li H. Expectation decreases brain susceptibility to fearful stimuli: ERP evidence from a modified emotion evaluation task. Neurosci Lett 2012; 514: 198-203, doi: 10.1016/j.neulet.2012.02.094.

13. Stewart JL, Silton RL, Sass SM, Fisher JE, Edgar JC, Heller $\mathrm{W}$, et al. Attentional bias to negative emotion as a function of approach and withdrawal anger styles: an ERP investigation. Int J Psychophysiol 2010; 76: 9-18.

14. Gootjes L, Coppens LC, Zwaan RA, Franken IH, Van Strien JW. Effects of recent word exposure on emotion-word Stroop interference: an ERP study. Int J Psychophysiol 2011; 79: 356-363.

15. Fields EC, Kuperberg GR. It's all about you: an ERP study of emotion and self-relevance in discourse. Neuroimage 2012; 62: 562-574, doi: 10.1016/j.neuroimage.2012.05.003

16. Xu Q, Yang $Y$, Wang $P$, Sun $G$, Zhao L. Gender differences in preattentive processing of facial expressions: an ERP study. Brain Topogr 2013; 26: 488-500, doi: 10.1007/s10548-0130275-0.

17. Lang PJ, Bradley MM, Cuthbert BN. Motivated attention: affect, activation, and action. In: Lang PJ, Simons RF Balaban MT (Editors), Attention and orienting: Sensory and motivational processes. New Jersey: Lawrence Erlbaum Associates, Inc., Publishers; 1997. p 97-135.

18. Carretie L, Mercado F, Tapia M, Hinojosa JA. Emotion, attention, and the 'negativity bias', studied through eventrelated potentials. Int J Psychophysiol 2001; 41: 75-85.

19. Herbert C, Kissler J, Junghofer M, Peyk P, Rockstroh B. Processing of emotional adjectives: Evidence from startle EMG and ERPs. Psychophysiology 2006; 43: 197-206, doi: 10.1111/j.1469-8986.2006.00385.x.

20. Patrick CJ. Psychophysiological correlates of aggression and violence: an integrative review. Philos Trans $R$ Soc Lond $B$ Biol Sci 2008; 363: 2543-2555, doi: 10.1098/rstb.2008.0028.

21. Huang YX, Bai L, Ai H, Li W, Yu C, Liu J, et al. Influence of trait-anxiety on inhibition function: evidence from ERPs study. Neurosci Lett 2009; 456: 1-5, doi: 10.1016/j.neulet. 2009.03.072.

22. Brown TA, DiNardo PA, Barlow DH. Anxiety disorders interview schedule for DSM-IV: lifetime version (ADIS-IV-L). New York: Oxford University Press; 1994.

23. Bai L, Ma H, Huang YX, Luo YJ. The development of native chinese affective picture system-a pretest in 46 college 
students. Chin Ment Health J 2005; 19: 719-722.

24. Wang YN, Zhou LM, Luo YJ. The pilot establishment and evaluation of Chinese affective words system. Chin Ment Health J 2008; 22: 608-612.

25. Moser JS, Hajcak G, Bukay E, Simons RF. Intentional modulation of emotional responding to unpleasant pictures: an ERP study. Psychophysiology 2006; 43: 292-296, doi: 10.1111/j.1469-8986.2006.00402.x.

26. Hjorth B. An on-line transformation of EEG scalp potentials into orthogonal source derivations. Electroencephalogr Clin Neurophysiol 1975; 39: 526-530, doi: 10.1016/0013-4694(75) 90056-5.

27. Dong G, Lu S. The relation of expression recognition and affective experience in facial expression processing: an event-related potential study. Psychol Res Behav Manag 2010; 3: 65-74, doi: 10.2147/PRBM.S9211.

28. Albert J, Lopez-Martin S, Carretie L. Emotional context modulates response inhibition: neural and behavioral data. Neuroimage 2010; 49: 914-921, doi: 10.1016/j.neuroimage. 2009.08.045.

29. Dugas MJ, Gagnon F, Ladouceur R, Freeston $\mathrm{MH}$. Generalized anxiety disorder: a preliminary test of a conceptual model. Behav Res Ther 1998; 36: 215-226, doi: 10.1016/S0005-7967(97)00070-3.

30. Huang YX, Luo YJ. Temporal course of emotional negativity bias: an ERP study. Neurosci Lett 2006; 398: 91-96, doi: 10.1016/j.neulet.2005.12.074.

31. Cisler JM, Koster EH. Mechanisms of attentional biases towards threat in anxiety disorders: An integrative review. Clin Psychol Rev 2010; 30: 203-216, doi: 10.1016/j.cpr.2009. 11.003.

32. Fox E, Cahill S, Zougkou K. Preconscious processing biases predict emotional reactivity to stress. Biol Psychiatry 2010; 67: 371-377, doi: 10.1016/j.biopsych.2009. 11.018.

33. Bradley BP, Mogg K, White J, Groom C, de Bono J. Attentional bias for emotional faces in generalized anxiety disorder. Br J Clin Psychol 1999; 38 (Part 3): 267-278.

34. Mennin DS, Heimberg RG, Turk CL, Fresco DM. Applying an emotion regulation framework to integrative approaches to generalized anxiety disorder. Clin Psychol-Sci Pr 2002; 9: 85-90, doi: 10.1093/clipsy.9.1.85.

35. Campbell-Sills L, Barlow DH, Brown TA, Hofmann SG. Effects of suppression and acceptance on emotional responses of individuals with anxiety and mood disorders. Behav Res Ther 2006; 44: 1251-1263, doi: 10.1016/j.brat.2005.10.001. 